\title{
Nonlinear route to intrinsic Josephson oscillations in spinor cavity-polariton condensates
}

\author{
S. S. Gavrilov, ${ }^{1}$ A. S. Brichkin, ${ }^{1}$ S. I. Novikov, ${ }^{1}$ S. Höfling, ${ }^{2,3}$ C. Schneider, ${ }^{2}$ M. Kamp, ${ }^{2}$ A. Forchel,,${ }^{2}$ and V. D. Kulakovskii ${ }^{1}$ \\ ${ }^{1}$ Institute of Solid State Physics RAS, 142432 Chernogolovka, Russia \\ ${ }^{2}$ Technische Physik, Physikalisches Institut and Wilhelm Conrad Röntgen Research Center for Complex Material Systems, \\ Universität Würzburg, D-97074 Würzburg, Germany \\ ${ }^{3}$ SUPA, School of Physics and Astronomy, University of St Andrews, St Andrews KY16 9SS, United Kingdom
}

(Received 29 July 2014; revised manuscript received 2 December 2014; published 9 December 2014)

\begin{abstract}
The intrinsic Josephson effect is observed in a two-component polariton condensate in a strained planar microcavity under a resonant pulsed excitation such that only one of the two linearly polarized eigenstates is pumped from the outside. On reaching the threshold density, the polariton-polariton interaction involves a spontaneous breaking of the symmetry between spin-up and spin-down polaritons. As a result, the condensate acquires nearly circular polarization while the pump is strong and then passes to the Josephson oscillation regime since the pump weakens.
\end{abstract}

DOI: 10.1103/PhysRevB.90.235309

PACS number(s): 71.36.+c, 71.35.Lk, 42.65.Pc

\section{INTRODUCTION}

In this work, we investigate the intrinsic Josephson effect in a dissipative condensate of exciton polaritons in a GaAs-based microcavity. Cavity polaritons are composite bosons formed due to the strong coupling between cavity photons and quantum well excitons [1]. A very small effective mass borrowed from the light mode $\left(\sim 10^{-5}\right.$ of the mass of free electrons) allows polaritons to undergo Bose-Einstein condensation at comparatively high temperatures of about $10 \mathrm{~K}$ in GaAs and CdTe cavities [2-4] and even $300 \mathrm{~K}$ in GaN cavities [5]. Both the amplitude and phase of a polariton condensate are easily probed by optical means as they are imprinted in the emitted light. On the other hand, emission out of the cavity involves a limited lifetime of polaritons (of about $10 \mathrm{ps}$ in GaAs cavities), which renders them out of equilibrium and results in their specific features under resonant excitation. Cavity polaritons are unique in that their macroscopically coherent (condensate) states can be created both in a quasiequilibrium way typical of cold atoms or under the strongly nonequilibrium conditions of resonant and coherent optical driving.

Collective phenomena that are very similar to the Josephson effect in superconductors [6] take place in both Bose-Einstein condensates of cold atoms [7-10] and macroscopically coherent cavity-polariton systems [11-15]. Two different effects are considered. The extrinsic Josephson effect comes from the tunnel coupling of the two condensates trapped in separate minima of the disorder potential; in the case of cavity polaritons, it was observed in CdTe microcavities [12]. By contrast, the intrinsic Josephson effect in Bose systems is related to the coupling between different spin components at the same space location [11].

Optically pumped polaritons have a total angular momentum (conventionally referred to as polariton spin) of $|\mathbf{J}|=1$. The two projections of $\mathbf{J}$ on the normal to the cavity plane, $J_{z}=$ \pm 1 , correspond to the right- and left-circular polarizations of photons. The polariton-polariton interaction is spin sensitive. Normally, polaritons with opposite spins are nearly uncoupled, but polaritons with the same spins strongly repel each other so that the condensate level experiences a blueshift that can largely exceed its linewidth [16-24]. The difference in the interaction strengths of same- and opposite-spin polaritons results in the multistability under resonant and coherent driving [25-32]. At the same time, spatial anisotropy of the cavity plane (e.g., due to a lateral strain) leads to the splitting of the orthogonally polarized polariton eigenstates. Although the effect of the splitting by itself is "linear," its combination with the repulsive pair interaction results in a spontaneously broken symmetry between opposite-spin states of the condensate, which is accompanied by a linear-to-circular polarization conversion under a strictly linearly polarized (symmetrical) excitation [33-35].

Consider the condensate in an anisotropic cavity in which the orthogonally polarized eigenstates are split in energy. The splitting yields coupling between opposite-spin components of the field, hence one expects the intrinsic Josephson oscillation to occur so long as both of the two eigenstates are excited. If, however, the pump is resonant and spectrally narrow and excites only one of the eigenstates, then the Josephson oscillations are impossible in the linear regime where no "tunneling" between the eigenstates is allowed. Here we report on the nonlinear mechanism which brings on the possibility for the oscillations to arise.

In this work, we evidence the intrinsic Josephson effect in a bosonic cavity-polariton system under a spectrally narrow resonant pulsed excitation. The pump polarization corresponds to the upper of the split lower polariton states and the pump frequency is slightly above the resonance level. The pump duration is about $100 \mathrm{ps}$ and its spectral width is $20 \mu \mathrm{eV}$, which is four times smaller than the splitting of the eigenstates $(\delta)$. A pronounced oscillation at frequency $\delta / h$ in the circular polarization of the condensate is observed at the pulse tail where the contribution from the pump source to the polariton density becomes negligible. This effect occurs in a threshold manner as it is mediated by a nonlinear process of a spontaneous breaking of the symmetry between opposite-spin polaritons.

Below, in Sec. II, we describe the sample and experimental setup. In Sec. III, we consider the evolution of the cavity signal intensity as well as its spectral composition and analyze the intrinsic Josephson oscillations. Finally, Sec. IV contains concluding remarks. 

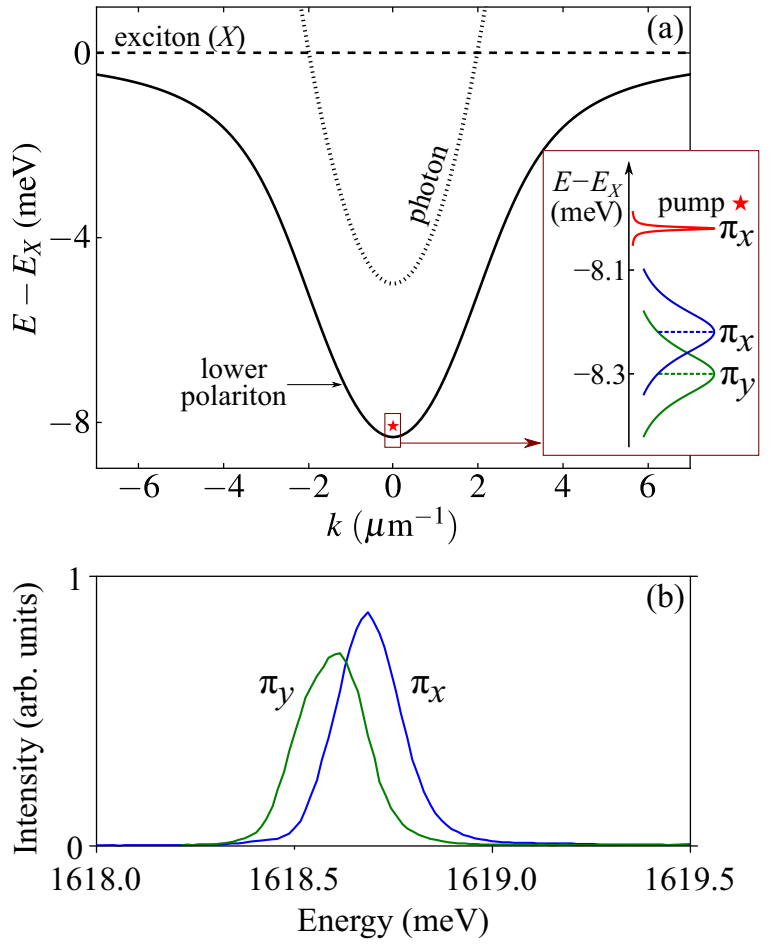

FIG. 1. (Color online) (a) Sketch of the cavity-polariton dispersion end excitation parameters. The free exciton level is $E_{X} \approx$ $1627 \mathrm{meV}$. Inset: Energies and polarizations of the polariton and 100 ps pump levels. (b) Transmission spectra measured under a wide-band 1-ps-long excitation (pulse FWHM was about $3 \mathrm{meV}$ ).

\section{EXPERIMENT}

The sample and experimental setup are the same as that used in Refs. [33,34]; for completeness, here we repeat their characteristics. The GaAs/AlAs microcavity is grown by molecular-beam epitaxy on top of a GaAs substrate. It consists of four 7-nm-thick GaAs quantum wells separated by 4-nm-thick AlAs barriers centered at antinodes of the electric field in a $\lambda / 2$ cavity. The top (bottom) mirror consists of 32 (36) $\mathrm{Al}_{0.2} \mathrm{Ga}_{0.8} \mathrm{As} / \mathrm{AlAs}$ Bragg reflectors. For transmission measurements, a $700 \times 300 \mu \mathrm{m}^{2}$ window was etched out of the GaAs substrate using citric acid/hydrogen peroxide selective etch (the AlAs layer in the bottom Bragg mirror served as the etch stop layer).

Figure 1(a) summarizes the parameters of the polariton dispersion law and excitation scheme. The bottom of the lower polariton branch is at $1.619 \mathrm{eV}$, and the $Q$ factor exceeds $10^{4}$; the Rabi splitting and the exciton-photon detuning at zero in-plane wave vector $(\mathbf{k}=0)$ are $\Omega \approx 10.5 \mathrm{meV}$ and $E_{C}-E_{X} \approx-5 \mathrm{meV}$, respectively. Under a weak wide-band excitation at $\mathbf{k}=0$, the cavity transmission spectrum consists of two orthogonally polarized $\pi_{x}$ and $\pi_{y}$ modes separated by $\delta=E_{x}-E_{y}=80 \pm 5 \mu \mathrm{eV}$ [Fig. 1(b)]. The ground state is split due to a lowered symmetry within the strained etched area. The eigenstates are polarized along the sides of the etched window; those, in turn, are parallel to the [110] and [1 $\overline{10}$ ] crystal axes referred to as $x$ and $y$.

Lower polariton states are excited by nearly transformlimited optical pulses with a duration of 100 ps and repetition rate of $8 \mathrm{MHz}$ generated by a mode-locked Ti:sapphire laser. The pump beam is directed along the cavity normal ( $z$ axis) and focused into a $40-\mu \mathrm{m}$-wide spot on the sample. The signal is collected from the center of that spot over a 4- $\mu$ m-wide area in which the pump density is nearly constant. The laser is detuned by $0.24 \mathrm{meV}$ above $\left(E_{x}+E_{y}\right) / 2$ at $\mathbf{k}=0$. The pump polarization is close to linear: its degrees of linear and circular polarizations are $\rho_{x, y}=\left(I_{p, x}-I_{p, y}\right) /\left(I_{p, x}+I_{p, y}\right) \approx$ +0.998 and $\rho_{c}=\left(I_{p}^{+}-I_{p}^{-}\right) /\left(I_{p}^{+}-I_{p}^{-}\right) \approx 0.05$, respectively, with $I_{p, x(y)}$ and $I_{p}^{ \pm}$being the pump intensities in the linear $\left[\pi_{x(y)}\right]$ and circular $\left[\sigma^{ \pm}\right]$polarization components. A polarizer and a $\lambda / 4$ wave plate are used to select a particular polarization component. The signal is recorded with a streak camera. All measurements are performed in an optical cryostat at $T=2 \mathrm{~K}$.

\section{RESULTS AND DISCUSSION}

Figure 2(a) shows the time dependences of the transmission signal recorded at the pump frequency as well as the spectrally

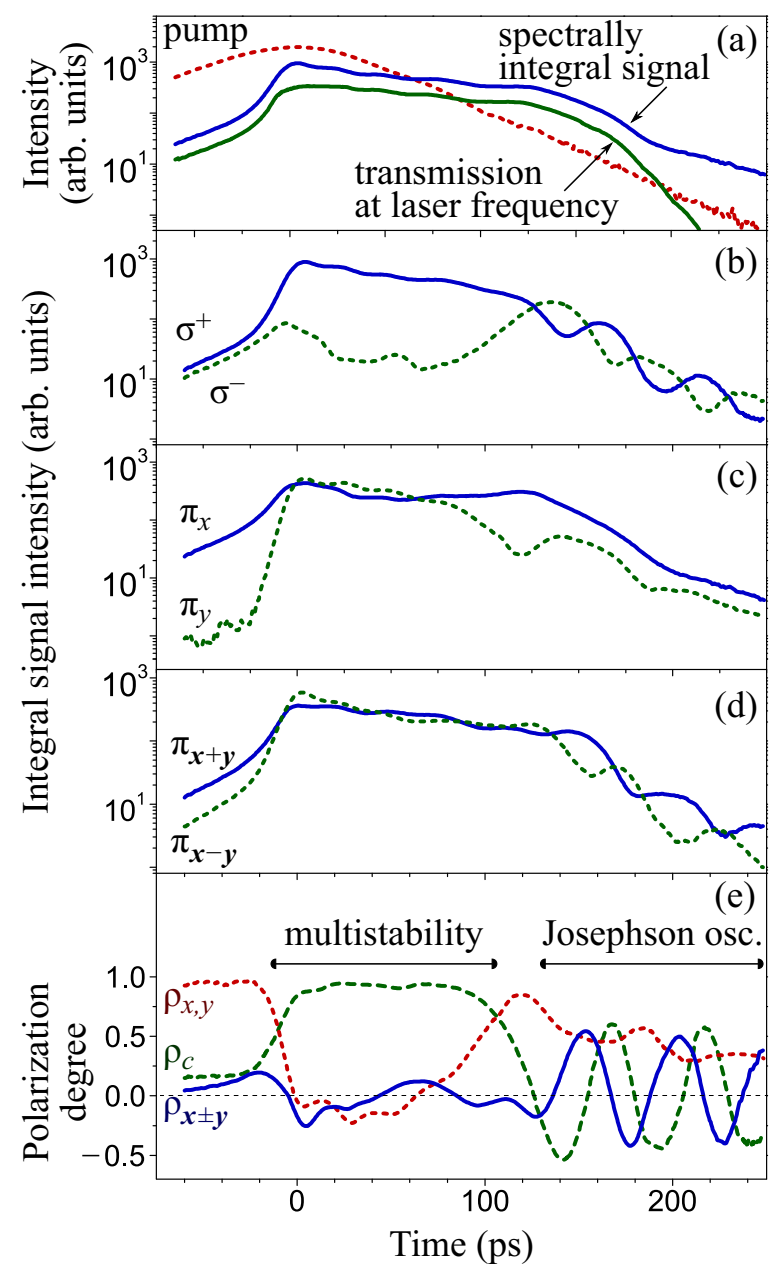

FIG. 2. (Color online) (a) Time dependences of the intensities of the pump, the transmission signal at the pump frequency, and the spectrally integral signal. (b)-(d) The intensities of the integral signal, recorded in (b) circular $\left(\sigma^{+}\right.$and $\left.\sigma^{-}\right)$, (c) linear $\left(\pi_{\mathbf{x}}\right.$ and $\left.\pi_{\mathbf{y}}\right)$, and (d) rotated linear $\left(\pi_{\mathbf{x}+\mathbf{y}}\right.$ and $\left.\pi_{\mathbf{x}-\mathbf{y}}\right)$ polarizations. (e) Corresponding degrees of the circular and two linear polarizations of the integral signal. 
integral signal intensity $I_{s}$ measured in the transmission geometry. Individual polarization components of the integral signal are shown in Figs. 2(b)-2(d). The corresponding polarization degrees (normalized Stokes parameters) $\rho_{a b}=\left(I_{s}^{(a)}-\right.$ $\left.I_{s}^{(b)}\right) /\left(I_{s}^{(a)}+I_{s}^{(b)}\right)$ are presented in Fig. 2(e). Shown are the circular polarization $\left(a, b=\sigma^{ \pm}\right)$and the linear polarizations in the base of the eigenstates $\left(a, b=\pi_{\mathbf{x}, \mathbf{y}}\right)$ and in the base rotated through $45^{\circ}$ relative to the latter $\left(a, b=\pi_{\mathbf{x} \pm \mathbf{y}}\right)$. The signals are recorded under normal incidence and an approximately $\pi_{x}$-polarized pump whose frequency is $0.2 \mathrm{meV}$ higher than that of the upper of the two lower polariton eigenstates that is polarized in the $x$ direction. The pulse profile $I_{p}(t)$ is also displayed in Fig. 2(a); the moment of $t=0$ corresponds to the pulse maximum.

The signal intensity $I_{S}$ is proportional to the cavity-field intensity. According to Fig. 2(a), $I_{s}$ shows a nearly linear growth with $I_{p}$ at $t<-30 \mathrm{ps}$. It is followed by a sharp amplification at $t \approx-20$ ps that is accompanied by a linearto-circular polarization conversion of the transmitted signal.

The jump in intensity is due to the positive feedback loop between the amplitude and effective resonance frequency of the pumped mode, which, in turn, occurs due to a repulsive polariton-polariton interaction that blueshifts the condensate level towards the pump frequency [25,36]. Under cw pump conditions, such effect results in the "S"-shaped dependence of intracavity field on the pump field and is referred to as polariton bi- or multistability. Only two steady-state branches of solutions exist under a linearly polarized pump as long as the opposite-spin states of the condensate are uncoupled. However, more complicated polarization behavior comes into existence via (i) a nonlinear mechanism brought on by the attractive interaction between opposite-spin polaritons [25,27] and/or (ii) the splitting of the polariton eigenstates due to a lifted degeneracy in spatially anisotropic cavities. In Ref. [33], it is shown that the combination of the repulsive interaction between same-spin polaritons and the splitting between orthogonally polarized eigenstates results in a spontaneously broken symmetry between the $\sigma^{ \pm}$components of the field and, hence, a linear-to-circular polarization conversion like that seen in Fig. 2 (note that no pair interaction between $\sigma^{+}$and $\sigma^{-}$components is required). Figure 3 sketches the steady-state response of the considered system under strictly linearly polarized excitation in the framework of the Gross-Pitaevskii equations; more details are found in Refs. [33,35]. The sign of polarization at the $\sigma$ branch is chosen spontaneously; however, even a small bias of the pump polarization to $\sigma^{+}$or $\sigma^{-}$ predetermines the same sign of intracavity-field polarization above the threshold.

The return transition from the high- to low-energy state occurs at $t>140 \mathrm{ps}$. Expectedly, the dynamics of the signal at the pulse tail differs from what could be expected within the steady-state approximation illustrated in Fig. 3. Figure 2 shows that both the signal-to-pump ratio $\left(I_{s} / I_{p}\right)$ and signal polarization do not restore their values typical of the pulse beginning. The signal decreases nearly exponentially with a decay time of $25 \pm 3$ ps that is equal to the polariton lifetime, and $I_{s} / I_{p}$ even at $t>200 \mathrm{ps}$ exceeds $I_{s} / I_{p}$ at $t=-50 \mathrm{ps}$ by more than 50 times. The signal demonstrates pronounced oscillations in its circular $\left(\rho_{c}\right)$ and linear $\left(\rho_{\mathbf{x} \pm \mathbf{y}}\right)$ polarization degrees that are displaced in phase relative to each other by

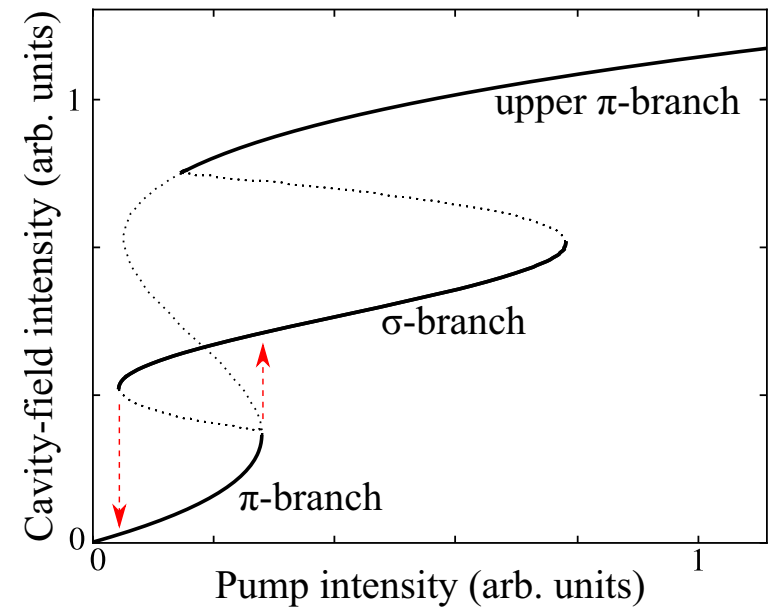

FIG. 3. (Color online) Steady-state response calculated within the one-mode approximation. Thin dotted lines show asymptotically unstable solutions. Dashed arrows indicate the $\pi$-to- $\sigma$ and reverse transitions that are considered in this work; the description of the transitions to the uppermost $\pi$ branch can be found elsewhere [33].

slightly less than one quarter of a period. Thus, at the pulse tail, the condensate state is no longer "driven" externallythe rapidly decreasing pump gets too weak for that-but is determined by the condensate's "own" parameters, the most important of which are the splitting of the eigenstates and the decay time.

Thus, with changing the pump density, the condensate passes through three qualitatively different stages of evolution. The first one is a nearly linear regime $(t<-30 \mathrm{ps})$. The second is the multistability period such that several macro-occupied states are possible at the same pump parameters and which of them is realized is spontaneously determined by fluctuations. Those states have opposite average spins close to \pm 1 , so that the symmetry between the $\sigma^{+}$and $\sigma^{-}$field components that is implied by a linearly polarized excitation gets broken on reaching the threshold amplitude. As the lifetime of polaritons is finite, the frequency of the intracavity field is expected to be pinned to the pump frequency while the pump is strong, which, however, is no longer the case at the pulse tail where the condensate evolves freely. At that (third) stage, the broken symmetry between $\sigma^{+}$and $\sigma^{-}$, which is inherited from the multistability period, manifests itself in a different manner and results in the Josephson oscillations. To clarify the transition from the multistability to the Josephson regime, below we analyze the evolution of the spectral composition of the signal.

Figure 4 shows the spectra recorded at several reference points: in the beginning of the pump pulse [Fig. 4(a)]; near the pulse maximum [Fig. 4(b)]; at the end of the multistability period [Fig. 4(c)]; and in the Josephson regime [Figs. 4(d) and 4(e)]. Approximate time and energy resolutions of the spectral measurements were $30 \mathrm{ps}$ and $60 \mu \mathrm{eV}$, respectively.

From the very beginning of the pulse and up to $t<100 \mathrm{ps,}$ the spectra exhibit only one peak that is located at the frequency of the pump wave $\left(E=E_{p}\right)$, which is characteristic of the case of resonant and coherent driving of a dissipative polariton state. The spectra recorded at $t=115,175$, and $215 \mathrm{ps}$, i.e., in the vicinity of and after the end of the multistability period, 


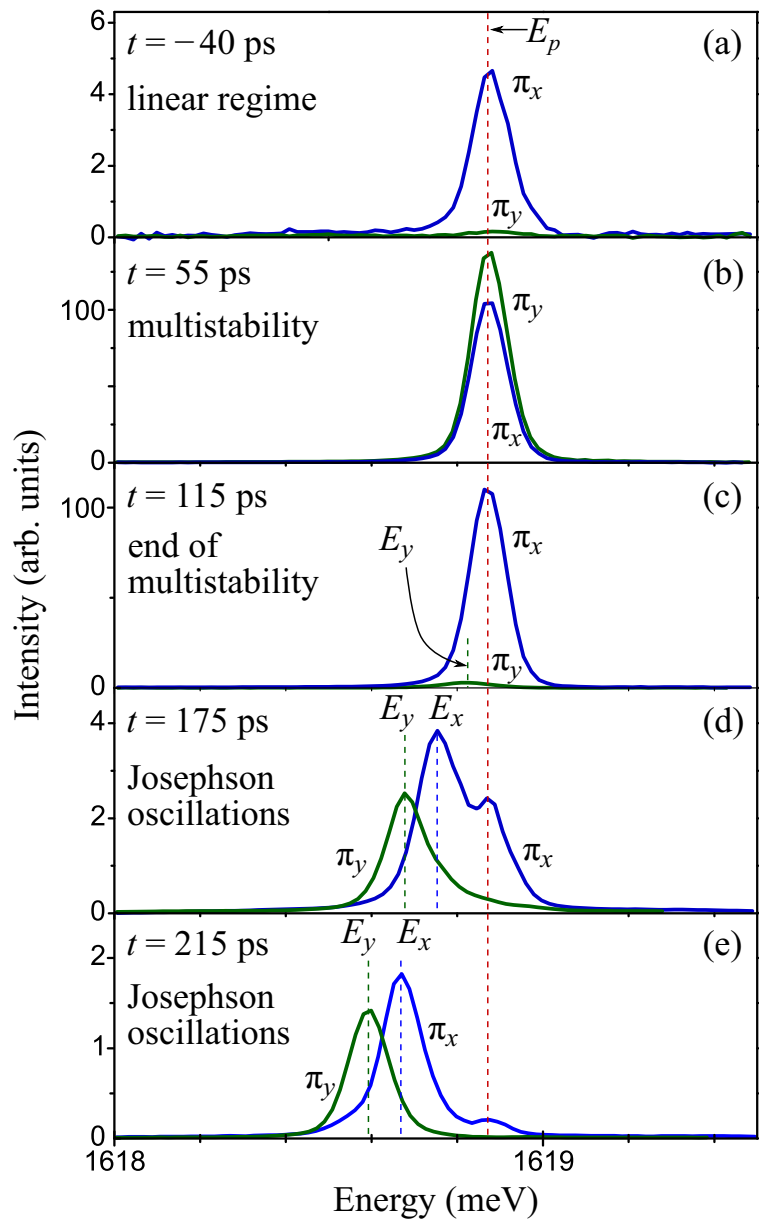

FIG. 4. (Color online) Signal spectra recorded in linear polarizations $\left(\pi_{\mathrm{x}}\right.$ and $\left.\pi_{\mathrm{y}}\right)$ at several reference time moments.

exhibit different features. Figure 4(c) shows that the $\pi_{x}$ and $\pi_{y}$ components of the intracavity field at $t=115 \mathrm{ps}$ become split in energy: the dominating $\left(\pi_{x}\right)$ polarization is still the strongest at $E=E_{p}$ whereas the peak of the minor one $\left(\pi_{y}\right)$, labeled as $E_{y}$, is redshifted by approximately $30 \mu \mathrm{eV}$. In addition to peaks $E_{p}$ and $E_{y}$, one more $\pi_{x}$-polarized line marked as $E_{x}$ appears at larger $t$ [Figs. 4(d) and 4(e)]. Eventually, peaks $E_{x}$ and $E_{y}$ tend to the eigenenergies of the $\pi_{x}$ - and $\pi_{y}$-polarized polariton levels.

The positions of the spectral maxima are shown in Fig. 5. In the interval $150<t<175 \mathrm{ps}$, the $\pi_{x}$-polarized lines $E_{p}$ and $E_{x}$ corresponding to the pump and polariton levels are calculated by fitting $I_{s}(E)$ by a sum of two Gaussian functions; estimated errors do not exceed several $\mu \mathrm{eV}$. At earlier times $(t<150 \mathrm{ps})$, the $\pi_{x}$-polarized polariton line cannot be distinguished from the pump line $E_{p}$, whereas at $t>175 \mathrm{ps}$ the two lines, $E_{x}$ and $E_{p}$, are resolved by the naked eye [Fig. 4(d)]. Figure 5 shows that the difference between the $\pi_{x}$ and $\pi_{y}$ energy levels grows quite rapidly at $120<t<140$ ps. Afterwards, difference $E_{x}-E_{y}$ remains nearly constant at $t>150 \mathrm{ps}$, resulting in a nearly constant Josephson oscillation period.

The signal at the pump frequency decreases much faster than the total signal, which is clearly seen in Figs. 4 and 2(a). The pump-frequency component becomes negligible by

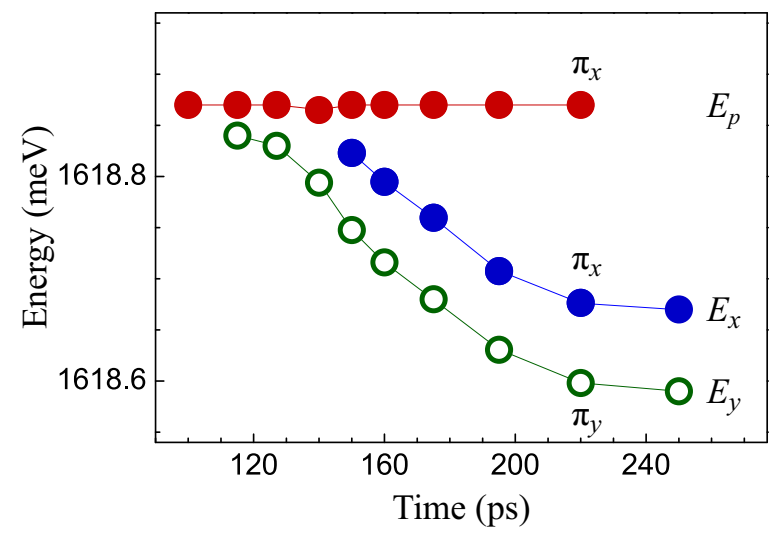

FIG. 5. (Color online) Energies of spectral lines at $t>100 \mathrm{ps}$.

$t>170$ ps. Thus, at the pulse tail, we deal with a freely decaying rather than pumped polariton condensate. It only can be slightly fed by a long-lived excitonic reservoir excited when the pump was strong [23,29,37,38]; however, this effect cannot be pronounced in our system where the pump is comparatively short term and acts far below the exciton level.

Figure 2(e) shows that the degree of linear polarization $\rho_{\mathbf{x}, \mathbf{y}}$ changes quite weakly at $t>180 \mathrm{ps}$. By contrast, $\rho_{\mathbf{x} \pm \mathbf{y}}(t)$ and $\rho_{c}(t)$ show pronounced oscillations, indicating a continuous time variation in the phase difference $(\Delta \phi \propto t)$ of the $\pi_{x}$ and $\pi_{y}$ components after their separation in energy. Figure 6 shows the degrees of polarization at $140<t<240 \mathrm{ps}$; it is seen that the two periods of oscillation nearly repeat one another in all polarization components. Full polarization $\rho=$ $\sqrt{\rho_{c}^{2}+\rho_{\mathbf{x}, \mathbf{y}}^{2}+\rho_{\mathbf{x} \pm \mathbf{y}}^{2}}$ is less than one because of fluctuations in the individual pump pulses whose repetition rate is $8 \mathrm{MHz}$ (at each $t$, the signal is in fact averaged over many of them). Slight fluctuations in pulse powers give slightly different durations of the multistability periods and, thus, different

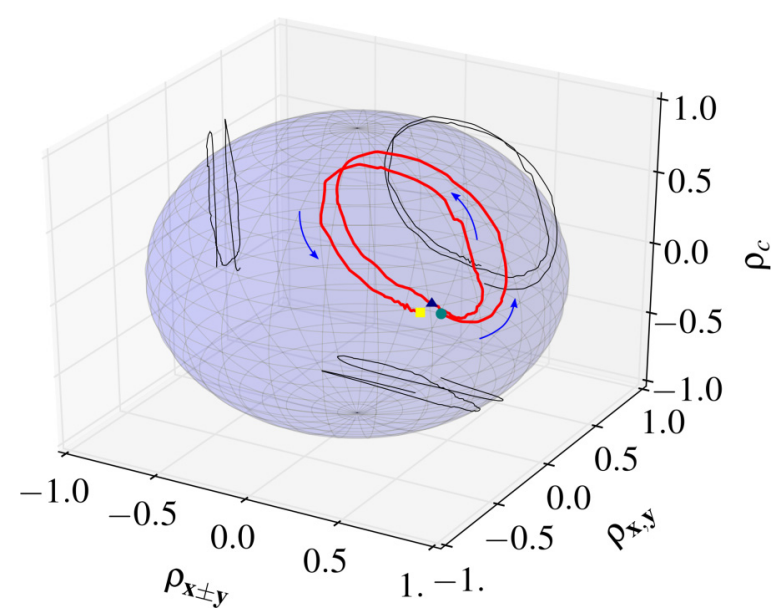

FIG. 6. (Color online) Degrees of polarization at $140<t<$ $240 \mathrm{ps}$ (heavy red line) and its projections on coordinate planes (thin black lines). Markers indicate the start (o), center $(t=190 \mathrm{ps}, \triangle)$, and end $(\square)$ points of the trajectory whose direction is indicated by blue curved arrows. The Poincaré sphere (shown for reference) has a radius of 1 . 
initial phases for the Josephson oscillations. Full polarization $\rho$ changes from $\sim 0.95$ at $t=120$ ps down to 0.8 at $t=140$ ps. Such changes can be considered small, thus, the experiment shows that a passage from the externally driven to freely decaying condensate state proceeds without a significant loss of coherence. Finally, $\rho$ reduces down to 0.6 by $t=240$ ps. Thus, the decoherence rate of a freely decaying condensate also appears to be quite small. It is significantly smaller than that of a quasiequilibrium condensate formed under a nonresonant excitation [39]. That is well expected as the decoherence in a resonantly pumped system is mainly due to the scattering on phonons, whereas nonresonantly pumped condensates lose their coherence through the interaction with an exciton reservoir that is inevitably excited in the latter case $[40,41]$.

The observed oscillations of $\rho_{\mathbf{x} \pm \mathbf{y}}$ and $\rho_{c}$ are the direct manifestation of the intrinsic Josephson effect in a coherent two-component condensate. The evolution of a free condensate is described by the Schrödinger equation with spin degrees of freedom,

$i \hbar \frac{\partial}{\partial t}\left(\begin{array}{c}\psi_{+} \\ \psi_{-}\end{array}\right)=\left[\hbar\left(\omega_{0}-i \gamma\right)-\frac{\hbar^{2} \nabla^{2}}{2 m}+\frac{\delta}{2}\left(\begin{array}{ll}0 & 1 \\ 1 & 0\end{array}\right)\right]\left(\begin{array}{l}\psi_{+} \\ \psi_{-}\end{array}\right)$,

where $\psi_{ \pm}$are the $\sigma^{ \pm}$components of a macroscopic wave function, $\hbar \omega_{0}=\left(E_{x}+E_{y}\right) / 2$ is the ground energy level, and $\gamma$ is the decay rate. Period $T$ of the oscillations is inversely proportional to splitting $\delta$, i.e., $T=h / \delta$, which is confirmed by Figs. 2 and 5 that reveal the independently measured values $T=50 \pm 3$ ps and $\delta=E_{x}-E_{y}=75 \pm$ $5 \mu \mathrm{eV}$.

Note that the oscillations can also be realized using a weak circular pump, provided its linewidth exceeds the splitting between the two eigenmodes so that both of them get actually excited. The role of multistability is that the same nearly full-range Josephson oscillations become possible irrespective of the spectral width and degree of circular polarization of the pump wave.

\section{CONCLUSION}

We have investigated the dynamics of a cavity-polariton condensate formed in a laterally strained cavity under pulsed resonant excitation. Although only one of the orthogonally polarized eigenstates is driven externally, a pronounced oscillation in the average spin of the condensate is observed at the tail of the pump pulse. Such oscillation is the direct manifestation of the intrinsic Josephson effect in a twocomponent polariton condensate [11].

The observed Josephson effect comes as a consequence of a two-stage process. At the first stage, the system is under a comparatively strong pump that compels macroscopic coherence of polaritons. Here the combination of the repulsive interpolariton interaction and the splitting of the eigenstates results in a spontaneously broken symmetry between opposite-spin polaritons under pumping with linearly polarized light $[33,35]$. As a result, intracavity field polarization gets converted from linear to almost circular well before the pump pulse density becomes weak. After the pulse is gone, the condensate persists owing to a comparatively long lifetime of polaritons and even much longer coherence time; it is no longer "driven" from the outside. At this stage, the splitting of the eigenstates manifests itself in the coupling between opposite-spin components of the condensate, yielding the intrinsic Josephson oscillations.

\section{ACKNOWLEDGMENTS}

We are grateful to N. A. Gippius and M. M. Glazov for fruitful discussions. This work was supported by the Russian Science Foundation (Grant No. 14-12-01372).
[1] C. Weisbuch, M. Nishioka, A. Ishikawa, and Y. Arakawa, Phys. Rev. Lett. 69, 3314 (1992).

[2] J. Kasprzak, M. Richard, S. Kundermann, A. Baas, P. Jeambrun, J. M. J. Keeling, F. M. Marchetti, M. H. Szymanska, R. André, J. L. Staehli, V. Savona, P. B. Littlewood, B. Deveaud, and L. S. Dang, Nature (London) 443, 409 (2006).

[3] R. Balili, V. Hartwell, D. Snoke, L. Pfeiffer, and K. West, Science 316, 1007 (2007).

[4] H. Deng, H. Haug, and Y. Yamamoto, Rev. Mod. Phys. 82, 1489 (2010).

[5] J. J. Baumberg, A. V. Kavokin, S. Christopoulos, A. J. D. Grundy, R. Butté, G. Christmann, D. D. Solnyshkov, G. Malpuech, G. Baldassarri Höger von Högersthal, E. Feltin, J.-F. Carlin, and N. Grandjean, Phys. Rev. Lett. 101, 136409 (2008)

[6] B. Josephson, Phys. Lett. 1, 251 (1962).

[7] S. Raghavan, A. Smerzi, S. Fantoni, and S. R. Shenoy, Phys. Rev. A 59, 620 (1999).

[8] F. S. Cataliotti, S. Burger, C. Fort, P. Maddaloni, F. Minardi, A. Trombettoni, A. Smerzi, and M. Inguscio, Science 293, 843 (2001).
[9] M. Albiez, R. Gati, J. Fölling, S. Hunsmann, M. Cristiani, and M. K. Oberthaler, Phys. Rev. Lett. 95, 010402 (2005).

[10] S. Levy, E. Lahoud, I. Shomroni, and J. Steinhauer, Nature (London) 449, 579 (2007).

[11] I. A. Shelykh, D. D. Solnyshkov, G. Pavlovic, and G. Malpuech, Phys. Rev. B 78, 041302 (2008).

[12] K. G. Lagoudakis, B. Pietka, M. Wouters, R. André, and B. Deveaud-Plédran, Phys. Rev. Lett. 105, 120403 (2010).

[13] D. Read, Y. G. Rubo, and A. V. Kavokin, Phys. Rev. B 81, 235315 (2010).

[14] C. Zhang and G. Jin, Phys. Rev. B 84, 115324 (2011).

[15] M. Abbarchi, A. Amo, V. G. Sala, D. D. Solnyshkov, H. Flayac, L. Ferrier, I. Sagnes, E. Galopin, A. Lemaitre, G. Malpuech, and J. Bloch, Nat. Phys. 9, 275 (2013).

[16] L. Viña, L. Muñoz, E. Pérez, J. Fernández-Rossier, C. Tejedor, and K. Ploog, Phys. Rev. B 54, R8317 (1996).

[17] M. Kuwata-Gonokami, S. Inouye, H. Suzuura, M. Shirane, R. Shimano, T. Someya, and H. Sakaki, Phys. Rev. Lett. 79, 1341 (1997).

[18] M. D. Martín, G. Aichmayr, L. Viña, and R. André, Phys. Rev. Lett. 89, 077402 (2002). 
[19] P. Renucci, T. Amand, X. Marie, P. Senellart, J. Bloch, B. Sermage, and K. V. Kavokin, Phys. Rev. B 72, 075317 (2005).

[20] K. V. Kavokin, P. Renucci, T. Amand, X. Marie, P. Senellart, J. Bloch, and B. Sermage, Phys. Status Solidi C 2, 763 (2005).

[21] I. A. Shelykh, A. V. Kavokin, Y. G. Rubo, T. C. H. Liew, and G. Malpuech, Semicond. Sci. Technol. 25, 013001 (2010).

[22] M. Vladimirova, S. Cronenberger, D. Scalbert, K. V. Kavokin, A. Miard, A. Lemaître, J. Bloch, D. Solnyshkov, G. Malpuech, and A. V. Kavokin, Phys. Rev. B 82, 075301 (2010).

[23] A. V. Sekretenko, S. S. Gavrilov, and V. D. Kulakovskii, Phys. Rev. B 88, 195302 (2013).

[24] N. Takemura, S. Trebaol, M. Wouters, M. T. Portella-Oberli, and B. Deveaud, Nat. Phys. 10, 500 (2014).

[25] N. A. Gippius, I. A. Shelykh, D. D. Solnyshkov, S. S. Gavrilov, Y. G. Rubo, A. V. Kavokin, S. G. Tikhodeev, and G. Malpuech, Phys. Rev. Lett. 98, 236401 (2007).

[26] I. A. Shelykh, T. C. H. Liew, and A. V. Kavokin, Phys. Rev. Lett. 100, 116401 (2008).

[27] S. S. Gavrilov, N. A. Gippius, S. G. Tikhodeev, and V. D. Kulakovskii, J. Exp. Theor. Phys. 110, 825 (2010).

[28] T. K. Paraïso, M. Wouters, Y. Léger, F. Morier-Genoud, and B. Deveaud-Plédran, Nat. Mater. 9, 655 (2010).

[29] D. Sarkar, S. S. Gavrilov, M. Sich, J. H. Quilter, R. A. Bradley, N. A. Gippius, K. Guda, V. D. Kulakovskii, M. S. Skolnick, and D. N. Krizhanovskii, Phys. Rev. Lett. 105, 216402 (2010).

[30] C. Adrados, A. Amo, T. C. H. Liew, R. Hivet, R. Houdré, E. Giacobino, A. V. Kavokin, and A. Bramati, Phys. Rev. Lett. 105, 216403 (2010).
[31] S. S. Gavrilov, A. S. Brichkin, A. A. Demenev, A. A. Dorodnyy, S. I. Novikov, V. D. Kulakovskii, S. G. Tikhodeev, and N. A. Gippius, Phys. Rev. B 85, 075319 (2012).

[32] Ö. Bozat, I. G. Savenko, and I. A. Shelykh, Phys. Rev. B 86, 035413 (2012).

[33] S. S. Gavrilov, A. V. Sekretenko, S. I. Novikov, C. Schneider, S. Höfling, M. Kamp, A. Forchel, and V. D. Kulakovskii, Appl. Phys. Lett. 102, 011104 (2013).

[34] A. V. Sekretenko, S. S. Gavrilov, S. I. Novikov, V. D. Kulakovskii, S. Höfling, C. Schneider, M. Kamp, and A. Forchel, Phys. Rev. B 88, 205302 (2013).

[35] S. S. Gavrilov, A. V. Sekretenko, N. A. Gippius, C. Schneider, S. Höfling, M. Kamp, A. Forchel, and V. D. Kulakovskii, Phys. Rev. B 87, 201303 (2013).

[36] A. Baas, J. P. Karr, H. Eleuch, and E. Giacobino, Phys. Rev. A 69, 023809 (2004).

[37] S. Gavrilov, A. Brichkin, A. Dorodnyi, S. Tikhodeev, N. Gippius, and V. Kulakovskii, J. Exp. Theor. Phys. Lett. 92, 171 (2010).

[38] M. Wouters, T. K. Paraïso, Y. Léger, R. Cerna, F. MorierGenoud, M. T. Portella-Oberli, and B. Deveaud-Plédran, Phys. Rev. B 87, 045303 (2013).

[39] V. V. Belykh, N. N. Sibeldin, V. D. Kulakovskii, M. M. Glazov, M. A. Semina, C. Schneider, S. Höfling, M. Kamp, and A. Forchel, Phys. Rev. Lett. 110, 137402 (2013).

[40] J. Schmutzler, T. Kazimierczuk, Ö. Bayraktar, M. Aßmann, M. Bayer, S. Brodbeck, M. Kamp, C. Schneider, and S. Höfling, Phys. Rev. B 89, 115119 (2014).

[41] V. V. Belykh and D. N. Sob’yanin, Phys. Rev. B 89, 245312 (2014). 